\title{
Fault Diagnosis with False and/or Missing Alarms in Distribution Systems with Distributed Generators
}

\author{
Guang Shen ${ }^{1}$, Yong Zhang ${ }^{2}$, Haifeng Qiu ${ }^{1}$, Chongyu Wang ${ }^{3}$, Fushuan Wen ${ }^{4,5, *}$, \\ Md. Abdus Salam ${ }^{6}$, Liguo Weng ${ }^{1}$, Bin Yu ${ }^{1}$ and Jie Chen ${ }^{1}$ \\ 1 State Grid Hangzhou Xiaoshan Power Supply Company, Beiganshan Road 12, Hangzhou 311201, China; \\ chen_bingfang@zj.sgcc.com.cn (G.S.); qiu_haifeng@zj.sgcc.com.cn (H.Q.); weng_liguo@zj.sgcc.com.cn (L.W.); \\ yu_bin_kj@zj.sgcc.com.cn (B.Y.); chen_jie_kj@zj.sgcc.com.cn (J.C.) \\ 2 State Grid Ningbo Power Supply Company, No. 1408 Liyuanbei Rd., Ningbo 315000, China; \\ yong.zhang1987@gmail.com \\ 3 School of Electrical Engineering, Zhejiang University, No. 38 Zheda Rd., Hangzhou 310027, China; \\ chongyu95@zju.edu.cn \\ 4 Department for Management of Science and Technology Development, Ton Duc Thang University, \\ Ho Chi Minh City, Vietnam \\ 5 Faculty of Electrical and Electronics Engineering, Ton Duc Thang University, Ho Chi Minh City, Vietnam \\ 6 Department of Electrical and Electronic Engineering, Universiti Teknologi Brunei, Bandar Seri Begawan \\ BE1410, Brunei; abdus.salam@utb.edu.bn \\ * Correspondence: fushuan.wen@tdtu.edu.vn; Tel.: +84-837-755-037; Fax: +84-837-755-055
}

Received: 18 July 2018; Accepted: 23 September 2018; Published: 27 September 2018

\begin{abstract}
A comprehensive method is presented in this work to locate faults in distribution systems with distributed generators (DGs). A two-level model is developed for this purpose with both telecommunication and telemetering data employed, so as to make good use of fused information for attaining a more credible optimization solution under scenarios with alarm distortions of feeder terminal units (FTUs) or loss during communication. First, at the upper level, an analytic model is developed to search all potential faulted sections/candidates based on the telecommunication data. Then, on the lower level, a model is presented using the telemetering data to identify the most likely fault location from the candidates provided by the upper model. The essential features of the two-level diagnosis model are demonstrated through a number of case studies. Simulation results have shown that the proposed approach is capable of not only locating the faulted section(s) in a distribution system with DGs but also identifying false and/or missing alarms.
\end{abstract}

Keywords: distribution system; fault location; distributed generator; two-level model

\section{Introduction}

Due to the limitation of transmission line corridors, distributed generators (DGs) are being increasingly installed in many countries and regions to fill the power supply deficiency. DGs are generally directly connected to the utility distribution feeders, which changes legacy distribution systems with unidirectional power flow to systems with multiple power sources and bidirectional power flow. Consequently, during fault conditions, the DGs on feeders will also contribute to the fault current with the contribution degree depending on the DGs' capacity, which may bring about significant impacts on protection schemes and fault diagnoses of distribution systems. In Reference [1], the conventional fault analysis method is extended to estimate fault current contributions under both balanced and unbalanced fault conditions. In Reference [2], a novel approach is proposed to study the impacts of DG penetration on recloser-fuse coordination based on a classification technique. In Reference [3], a new inverse-type relay is proposed to protect a distribution network with multiple 
DGs by accommodating changes in the fault current levels and reverse power flow. In Reference [4], models of protection devices and the general diagnostic engine (GDE) are presented, and the model is able to diagnose the malfunctioning of protection devices. In Reference [5], a grounded fault analysis method for microgrids is presented, and the impacts of a battery energy storage system (BESS) on grounded faults are discussed. In Reference [6], a new asymmetrical fault analysis method for microgrids with hybrid compensation is proposed and is applicable for single and simultaneous unsymmetrical faults. In Reference [7], a fault section diagnosis method for distribution substations is presented by employing fuzzy reasoning and rule matrix transformations. In Reference [8], a new power system fault diagnosis approach is presented, with the protection philosophy and the operation states of protective devices are taken into account.

Another advent intelligent technology is the distribution feeder automation (DFA) that has been widely implemented to meet the ever-increasing requirements for power quality and reliability. The DFA can be divided into two types according to the devices installed in the distribution system. One type is based on reclosers, fuses, and relays, while the other is based on a master station (MS), remote controllable switches (RCSs), and feeder terminal units (FTUs). A typical integrated DFA system in China is shown in Figure 1, which includes all the components of a scheme in the second type. The MS gathers information from the FTUs and other electric devices at regular intervals and sends opening or closing commands to the RCSs.

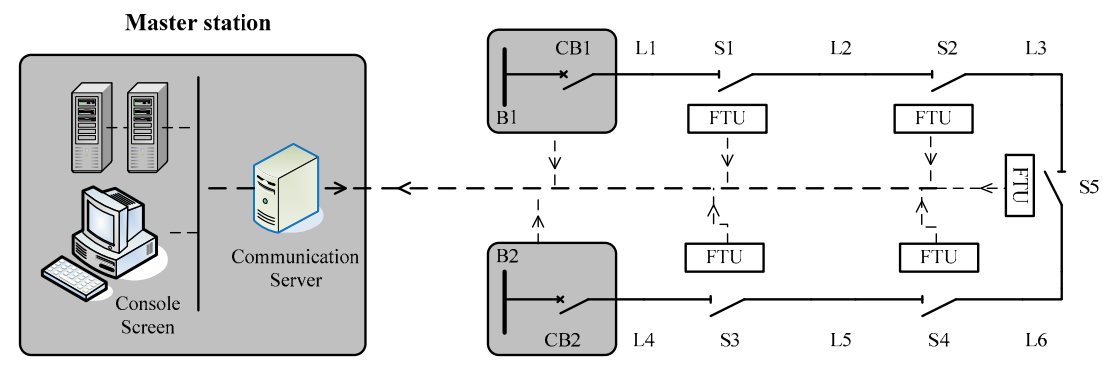

Figure 1. An integrated DFA system.

Among all the functions of a DFA, determining a fault location is regarded as the most important one. In a second type DFA scheme, an FTU can provide much richer remote information related to a fault location. Nevertheless, those FTUs are usually installed in outdoor and some other poor environments vulnerable to various communication interferences. To locate faulted sections in a distribution system using FTUs, as a result, one needs to thoroughly take into account all the uncertain factors such as potential false states and/or missing signals of FTUs.

Substantial research has been carried out in distribution system fault diagnosis, and various models and algorithms have been proposed, including integrating available pieces of probability-based evidence for fault diagnosis [9], using measurements from intelligent electronic devices (IEDs) for locating faults [10], comparing the interrupted load with the actual load in each line for identifying faults [11], analyzing the three-phase circuit to locate faults in an unbalanced distribution system [12], and employing the wavelet-based artificial neural network for fault analysis [13]. Nevertheless, most of these fault-locating methods are for radial systems with a single source and are not applicable to distribution systems with DGs [14]. Hence it is necessary to develop new models and algorithms.

Current methods to trace faults in distribution systems with DGs fall into two categories. One is the matrix-based algorithm [15,16] and the other is based on electrical data [2]. By analyzing the characteristics of fault currents, the matrix-based algorithm constructs the fault judgment matrix to identify the fault section. This algorithm makes use of only the telecommunication data from FTUs and may suffer insufficient or misleading information problems when experiencing communication network mistakes. Obviously, the deduced fault judgment matrix would then be unable to reflect the actual fault conditions and thereby obtained fault location could be inaccurate. The second category takes the current and voltage measurements all over the system to identify and isolate the faulted 
section. This approach, which relies on the DFA system equipped with reclosers, fuses, and relays, performs the diagnosis of the fault section using only the telemetering readings.

Given the above stated background, an FTU-based method is proposed in this paper to locate faults in $35 \mathrm{kV} / 10 \mathrm{kV}$ distribution systems with DGs. Aimed at an integrated DFA system as shown in Figure 1, the new method can improve the reliability of the fault diagnosis by virtue of redundancy of the exploited telecommunication and telemetering data, even though false and/or missing alarms during the communication process may occur from time to time. Since it is unlikely for multiple faults to occur on one feeder simultaneously, this paper studies the cases where only a single fault may occur on one feeder at a specified time point [17]. The paper is organized as follows. Section 2 outlines the two-level theoretical model of the method. In Section 3, a sample system is served for demonstrating the essential features of the developed diagnosis scheme in locating a fault of a distribution system with DGs. Lastly, Section 4 presents concluding remarks.

\section{Model for Locating Faults in Distribution Systems with DGs}

This section discusses the two-level model developed for locating faults in distribution systems with DGs. The framework of such a model is illustrated in Figure 2. First, an analytical model is built up at the upper level based on the telecommunication data and the genetic algorithm (GA) is used to obtain the candidate hypotheses of the faulted devices. The model at the lower level is based on the telemetering data to filter out the faulted section(s) from the hypotheses provided by the upper level. An abrupt variation of the current magnitude is taken as a criterion in this process. The presented two-level model can not only diagnose the faulted section(s) by using both telecommunication data and telemetering data but also identify the incorrect and missing alarm messages.

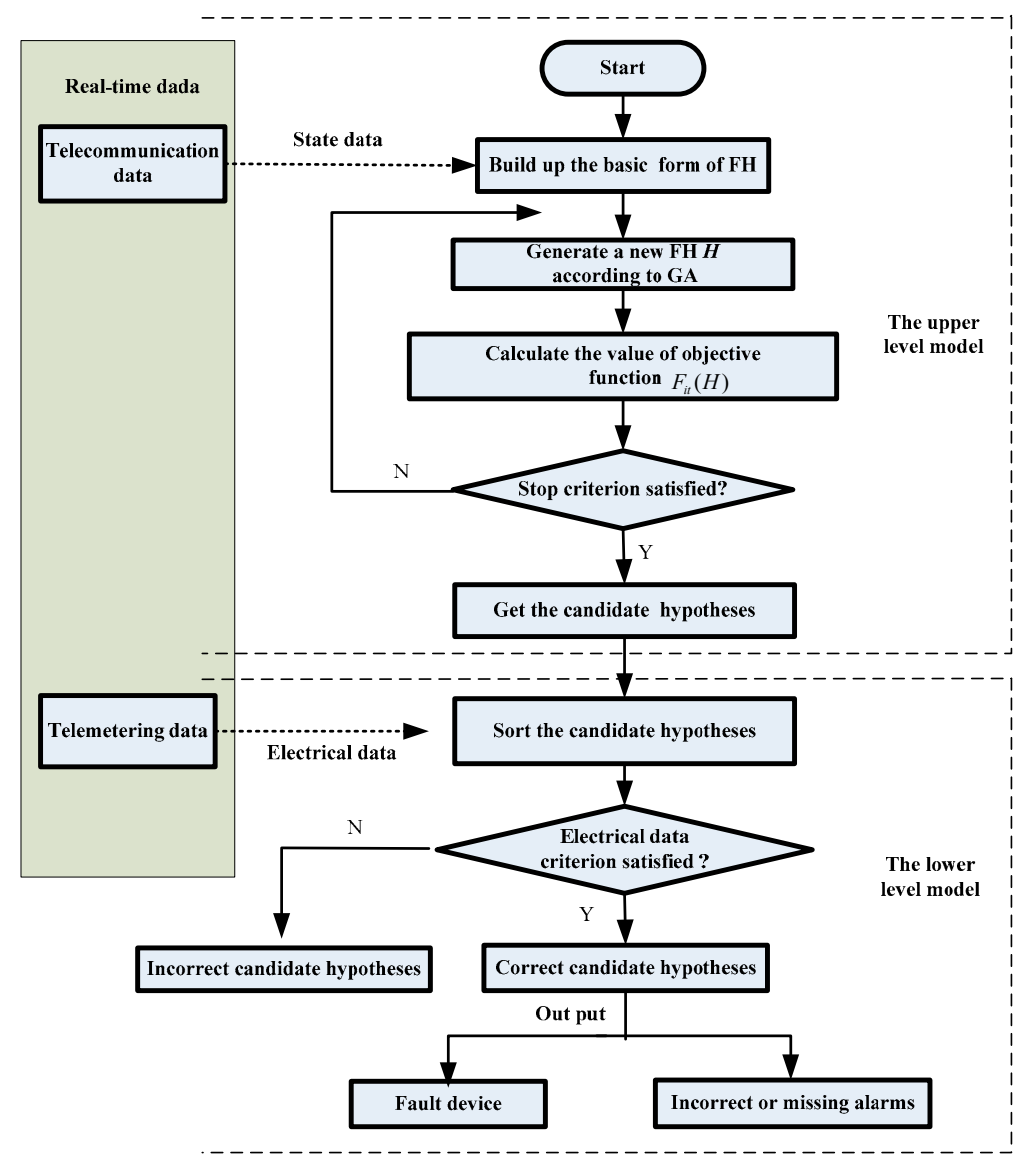

Figure 2. The framework of the developed fault location model. 


\subsection{The Analytic Model at the Upper Level}

The main design of the analytic model at the upper level is to formulate an optimization problem with the objective function reflecting the discrepancy between the actual and the expected alarm messages. The fault location in distribution systems is thus described by such an unconstrained 0-1 integer programming problem and the GA is exploited to search for a fault hypothesis $(\mathrm{FH})$ or hypotheses (FHs) which could well explain the received alarm messages [18,19].

In the analytic model searching for the fault location, each of the sectionalizing switches, the tie switches, and the inbound circuit breakers is viewed as a node, and every transmission line between the nodes is regarded as an independent device. In the DFA system, each node is equipped with an FTU to acquire the telecommunication, telemetering, telecontrol, and teleregulation data. Whenever there is a fault, each FTU will upload the state change information of the switch and collect the fault information including the magnitude and the direction of the fault current and voltage, and the fault temporal data [20]. Once the detected current exceeds a preset value $I_{\mathrm{Jzd}}$, the corresponding FTU will treat it as a fault current, with the FTU state change from 0 to 1 being reported to the MS. To avoid false operation of the FTU, $I_{\mathrm{Jzd}}$ should be set larger than the maximum load current of the system. More details are further discussed below, including the FHs, the objective function, the expected state of the sectionalizing switch, and the solving algorithm of the GA-based analytic model.

\subsubsection{The Fault Hypotheses (FHs)}

The following are the three vectors forming a fault hypothesis:

1. $\quad \boldsymbol{D}=\left[d_{1}, d_{2}, \ldots, d_{k}, \ldots, d_{n}\right]$ is the set of device states, where $n$ is the total number of devices of the distribution system, $d_{k}$ represents the state of the $k$ th device, with $d_{k}=1$ and $d_{k}=0$ corresponding to the fault and the normal state, respectively;

2. $L=\left[l_{1}, l_{2}, \ldots, l_{k}, \ldots, l_{n}\right]$ is the set of missing states of the FTUs, with $l_{k}=1$ and $l_{k}=0$ corresponding to the missing and the normal state of the $k$ th FTU, respectively;

3. $\boldsymbol{W}=\left[w_{1}, w_{2}, \ldots, w_{k}, \ldots, w_{n}\right]$ is the set of false states of the FTUs, with $w_{k}=1$ and $w_{k}=0$ corresponding to the false and normal state the $k$ th FTU, respectively.

By taking into account missing and false alarms from FTUs, an FH can be expressed as

$$
H=[D, L, W]
$$

Logically, each FH can be easily identified as either a reasonable FH or a contradictory FH. While a reasonable FH could potentially match the real fault, a contradictory FH should be excluded as a candidate due to its inconsistency. For example, it is impossible to have the missing state and the false state simultaneously with the same FTU as a contradictory FH might suggest [21]. When constructing the function to calculate the expected state of a sectionalizing switch, therefore, the logical rationality of the FH should be scrutinized to eliminate all the contradictory ones.

\subsubsection{The Objective Function}

The formulation of the objective function $F_{i t}(H)$ will directly affect the accuracy of the diagnosis regarding to the fault location. As discussed earlier, the fault locating is modeled into an unconstrained $0-1$ integer programming problem to minimize the objective function $F_{i t}(H)$, which is defined as the discrepancy between the actual and expected FTU states:

$$
F_{i t}(H)=\sum_{j=1}^{N}\left|I_{j}-I^{*}(H)\right|
$$

where $I_{j}$ and $I^{*}{ }_{j}(H)$ are the actual and expected states, respectively, of the sectionalizing switch corresponding to the $j$ th FTU. 
We have pointed out that the flow of distribution systems will change from unidirectional to bidirectional due to the connected DGs. Therefore, the power flow from the original power source to the loads and/or the DGs is defined as the positive direction as shown in Figure 3, which makes it insensitive to the capacity and the access points of the DGs when detecting the positive direction during a fault, and thereby improves the reliability of fault location diagnosis. By considering the influence of the connected DGs, $I_{j}$ is determined as follows:

$$
I_{j}=\left\{\begin{array}{cc}
1 & \text { the detected current exceeds } \mathrm{I}_{\mathrm{jzd}} \\
& \text { and is in the positive direction }
\end{array}\right.
$$

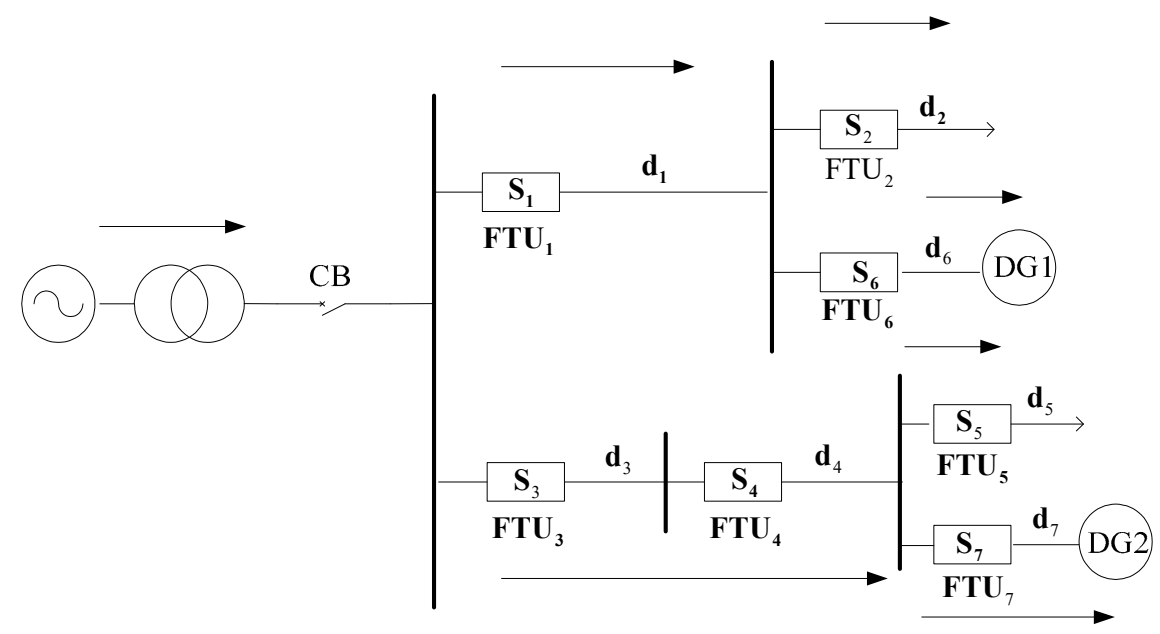

Figure 3. The structure of a DFA with two DGs using seven FTUs.

\subsubsection{The Function for Evaluating Expected States of Sectionalizing Switches}

According to the literature survey on fault location diagnosis in distribution systems, the expected states of sectionalizing switches are traditionally determined by the received device information without considering uncertainty factors like missing and/or false states of FTUs [1].

For convenience, in this paper, the expected states of sectionalizing switches are replaced by that of the corresponding FTUs. In the studied distribution system, the related-device set is defined as all the downstream components (along the positive direction) of the sectionalizing switch. Any faults of the related devices will, as expected, set the state of FTU to 1 providing that the FTU is in normal operation. Therefore, $I^{*}{ }_{j}(H)=\sum d_{i}$, where $d_{i}$ represents the $i$ th device in the related-device set of the $j$ th sectionalizing switch. Figure 3 shows the structure of a distribution system with DGs, and the related-device set of each sectionalizing switch is listed in Table 1.

Table 1. Relationship between sectionalizing switches and devices.

\begin{tabular}{cc}
\hline Sectionalizing Switch & Related-Device Set \\
\hline$S_{1}$ & $\left\{d_{1}, d_{2}, d_{6}\right\}$ \\
$S_{2}$ & $\left\{d_{2}\right\}$ \\
$S_{3}$ & $\left\{d_{3}, d_{4}, d_{5}, d_{7}\right\}$ \\
$S_{4}$ & $\left\{d_{4}, d_{5}, d_{7}\right\}$ \\
$S_{5}$ & $\left\{d_{5}\right\}$ \\
$S_{6}$ & $\left\{d_{6}\right\}$ \\
$S_{7}$ & $\left\{d_{7}\right\}$ \\
\hline
\end{tabular}


In the analytic model of the upper level, the expected state of an FTU is computed by taking into consideration the missing and/or false states of the FTU. Table 2 is the truth table of the actual status of the FTU referring to sectionalizing switch $s_{j}$.

Table 2. The truth table of actual states of FTUs.

\begin{tabular}{|c|c|c|c|c|}
\hline$l_{j}$ & $w_{j}$ & $\sum d_{i}$ & $I^{*}{ }_{j}(H)$ & Notes \\
\hline 0 & 0 & 0 & 0 & $\begin{array}{l}I^{*}(H)=0 \text { : all the related devices of } s_{j} \text { are normal, and the corresponding } \\
\text { FTU of } s_{j} \text { is working properly. }\end{array}$ \\
\hline 0 & 0 & 1 & 1 & $\begin{array}{l}I^{*}{ }_{j}(H)=1 \text { : at least one device in the related-device set of } s_{j} \text { is faulty, } \\
\text { and the corresponding FTU is working properly. }\end{array}$ \\
\hline 0 & 1 & 0 & 1 & $\begin{array}{l}I^{*}{ }_{j}(H)=1 \text { : all the related devices of } s_{j} \text { are normal, the alarm state should } \\
\text { be } 0 . \text { However, the corresponding FTU of } s_{j} \text { uploads a false alarm. }\end{array}$ \\
\hline 0 & 1 & 1 & $x$ & $\begin{array}{l}\left.I_{j}^{*}(H)=\mathrm{X} \text { (a contradictory } \mathrm{FH}\right) \text { : at least one device in the related-device } \\
\text { set of } s_{j} \text { is faulty, and thus it is logically impossible for the corresponding } \\
\text { FTU to upload a false alarm. }\end{array}$ \\
\hline 1 & 0 & 0 & $x$ & $\begin{array}{l}I^{*}{ }_{j}(H)=\mathrm{X} \text { : all the related devices of } s_{j} \text { are normal, and thus it is logically } \\
\text { impossible for the corresponding FTU to be missing an alarm. }\end{array}$ \\
\hline 1 & 0 & 1 & 0 & $\begin{array}{l}I^{*}{ }_{j}(H)=0 \text { : at least one device in the related-device set of } s_{j} \text { is faulty; thus, } \\
\text { the state of FTU should be } 1 \text { but the corresponding FTU failed to issue } \\
\text { an alarm }\end{array}$ \\
\hline 1 & 1 & 0 & $x$ & $\begin{array}{l}I^{*}{ }_{j}(H)=\mathrm{X} \text { : it is logically impossible to have a missing and false alarm } \\
\text { occurred at an FTU simultaneously. }\end{array}$ \\
\hline 1 & 1 & 1 & $x$ & $\begin{array}{l}I^{*}{ }_{j}(H)=\mathrm{X} \text { : it is logically impossible to have a missing and false alarm } \\
\text { occurred at an FTU simultaneously. }\end{array}$ \\
\hline
\end{tabular}

By analyzing the logics of the truth table, the expected state of the sectionalizing switch can be computed using:

$$
I^{*}{ }_{j}(H)=\sum d_{i} \otimes \overline{l_{j}} \otimes \overline{w_{j}} \oplus \overline{\sum d_{i}} \otimes \bar{l}_{j} \otimes w_{j}
$$

Equation (4) consists of two components $\sum d_{i} \otimes \overline{l_{j}} \otimes \overline{w_{j}}$ and $\overline{\sum d_{i}} \otimes \overline{l_{j}} \otimes w_{j}$, with the former reflecting the case that has at least a fault in the related-device set of $s_{j}$ and the FTU is working properly, and the latter reflecting the case that has no faults in the related-device set of $s_{j}$ but the FTU is not working properly. Both cases will lead to $I^{*}{ }_{j}(H)=1$.

The contradictory FHs, after further analysis, can be grouped into three types labeled with $\mathrm{X}$ as shown in Table 2. At the upper level, if $H$ is a contradictory one, $F_{i t}(H)$ should be assigned a large value, say, $F_{i t}(H)=10,000$, so that they will be weeded out quickly in the iterative process of minimizing the objective function.

\subsubsection{The Solving Algorithm}

At the upper level, the optimization problem described by Equation (2) can be solved using GA techniques. The optimizing procedures are summarized below.

Since it is difficult to determine a unique optimal solution due to the existence of uncertain factors, a group of candidates for the optimal solution could be kept during the course of the optimization. In each generation of the GA, the chromosome(s) corresponding to the minimum value of the objective function is (are) recorded. When a better objective function value is obtained in a new generation, the corresponding chromosome(s) is (are) recorded to replace the original one. Otherwise, the record is unchanged. In this way, the final solution could be a single or a group of chromosomes, known as the elitist strategy of GA [18].

Power system fault diagnosis is limited to the devices in the outage part of the power system concerned. Since the number of these devices is usually small, GA is fast enough for on-line fault 
diagnosis applications. The use of GA only provides a solution method to the presented fault diagnosis model. Certainly, other options exist for solving the problem.

\subsection{The Lower Level Model: Locating a Fault Based on Electrical Data}

Existing matrix-based optimization algorithms for locating faults in a distribution system employ only the telecommunication data without sufficient information redundancy while there are actually more information sources available. Aside from the telecommunication data, i.e., the state data, the telemetering data (electrical data) can also be exploited. Using both of them can definitely improve the accuracy of fault location diagnosis for distributed systems vulnerable to false and/or missing alarms of FTUs.

\subsubsection{The Concept of Stiffness Ratio}

The penetration of DGs in distribution systems would cause variation in electrical data. The stiffness ratio is introduced for the convenience in studying this influence. According to IEEE Standard 1547.2-2008, the stiffness ratio $\left(S_{t}\right)$ is defined as "the relative strength of the area EPS (electric power system) at the point of common coupling (PCC) compared with the DR (distributed resource), expressed in terms of the short-circuit kilovolt amperes of the two systems, calculated at the PCC". Here the DR is short for distributed resources, including both distributed generators and energy storage devices. Thus, $S_{t}$ is calculated using:

$$
S_{t}=\frac{S_{e p s}+S_{D G}}{S_{D G}}
$$

where $S_{e p s}$ is the short-circuit contribution in kVA from all other power sources in the area EPS and $S_{D G}$ is the short-circuit contribution in kVA from all the DGs [17].

\subsubsection{A Model to Locate a Fault Based on Electrical Data}

The candidate FHs obtained from the upper level analytic model are sorted according to the following rule of thumb: (1) Put the FHs in an order determined by the principal priority factor, i.e., the number of the faulted devices. The smaller the number is, the higher priority the corresponding FH becomes (an earlier position in the list). The FH with no faulty devices, however, should be placed at the end. (2) For the FHs with the same number of faulted devices, the second priority factor is applied. A larger sum of false and missing alarms leads to a lower priority. (3) To break the tie of (1) and (2), the number of missing alarms will be employed. In general, a missing alarm is more likely to occur than a false alarm is according to the statistics, thus the FH corresponding to a larger number of missing alarms is regarded as having a higher priority. Through sorting the FHs in accordance with the priorities of all fault scenarios, the lower level model could be improved significantly in terms of the accuracy and efficiency in searching for the optimal FH.

From the geographical location point of view, the fault in a distribution system can be divided into two types: (1) a fault at the T-point, i.e., at the terminal of a feeder; or (2) a fault occurred between the T-point and the incoming circuit breaker, the middle of a feeder.

1. A fault at the terminal T-point

When a fault occurs at the terminal T-point, i.e., at one of the three devices constructing the terminal, the FTU installed at the main power source side will detect the fault overcurrent. Consequently, the faulted section could be identified by analyzing the abrupt variation of current magnitude at the three related FTUs.

Take the system shown in Figure 3 as an example, where the terminal T-point consists of $d_{4}, d_{5}$, and $d_{7}$. If there is a fault at this terminal T-point, the detected current from the $\mathrm{FTU}_{4}$ will exceed the preset $I_{\mathrm{Jzd}}$. Then, currents from the $\mathrm{FTU}_{5}$ and $\mathrm{FTU}_{7}$ are further checked to find whether there is an 
abrupt current change. If the current magnitude monitored by $\mathrm{FTU}_{5}$ (or $\mathrm{FTU}_{7}$ ) exceeds $2 I_{\mathrm{Jzd}}$, then the faulted section is $d_{5}$ ( or $d_{7}$ ). In either case, the voltages monitored by each FTU present a monotonic decrease along the positive direction, which could be exploited as the second criterion for locating the fault. If neither of FTU ${ }_{5}$ and $\mathrm{FTU}_{7}$ detects a sudden current change $\left(>2 I_{\mathrm{Jzd}}\right), d_{4}$ is identified as the faulted section.

2. A fault at the middle section of a feeder

When a fault occurs at the middle of a feeder, the current magnitudes detected at the two sides of a healthy device are approximately the same, meanwhile the ratio between the absolute current detected by the upstream and the downstream FTUs of the faulted device exceeds a certain given threshold:

$$
\left|\frac{I_{d i k-1}}{I_{d i k}}\right|>k_{y z}
$$

where $I_{d i k-1}$ and $I_{d i k}$ are the upstream and the downstream fault currents, respectively, of device $d_{i}$ detected by $\mathrm{FTU}_{i}$, and $k_{y z}$ is the specified threshold.

Because in terms of the short-circuit capacity, the main power source is usually much larger than the DGs [17] (e.g., with a stiffness ratio $S_{t} \geq 20$ ), the contribution from the system side to the fault current is, in general, one order of magnitude larger than that from the DG side. Thus, the faulted section could be determined by using Equation (6), where $k_{y z}$ is related to the actual $S_{t}$ of the distribution systems. For instance, if $S_{t}=20$, the ratio $S_{e p s} / S_{D G}=19$, giving $k_{y z}=19$. Considering the impacts of the load current and the feature of the impedance, a certain margin should be maintained in setting $k_{y z}$.

\section{Application Examples}

The distribution system shown in Figure 3 is adopted as the study system to demonstrate performance of the developed two-level model.

At the upper level, the related parameters of GA are set as population size pops $=100$, maximum permitted iteration number $M G=1000$, crossover probability $\mathrm{Rc}=0.3$, and mutation probability $\mathrm{Rm}=0.6$. At the lower level, the system model for the $10-\mathrm{kV}$ level is built up using the GRS toolboxes developed by the Join Bright Company in Beijing, China. The related parameters are specified as: the transformer capacity is $31.5 \mathrm{MVA}$, the apparent power demands of Load 1 and Load 2 are respectively 5.9 MVA and 5 MVA, and the capacities of DG1 and DG2 are both 1.5 MVA. Besides, the parameters of feeders are given in Table 3 where the threshold $k_{y z}$ is set to 8 . In Table $3, R_{1}$ and $R_{0}$, and $X_{1}$ and $X_{0}$, are, respectively, the positive and zero sequence resistance and reactance.

Table 3. Parameters of the feeders (LGJ-240).

\begin{tabular}{cccc}
\hline & & Unit Resistance $(\Omega / \mathbf{k m})$ & Unit Resistance $(\Omega / \mathbf{k m})$ \\
\cline { 3 - 4 } Feeders & Length $(\mathbf{k m})$ & {$\left[\mathbf{R}_{\mathbf{1}} ; \mathbf{R}_{\mathbf{0}}\right]$} & \\
\hline$d_{1}$ & 4 & & \\
$d_{2}$ & 2 & & \\
$d_{3}$ & 3 & & \\
$d_{4}$ & 1.5 & {$[0.1313 ; 0.3743]$} & \\
$d_{5}$ & 1 & & \\
$d_{6}$ & 3 & & \\
$d_{7}$ & 1.5 & & \\
\hline
\end{tabular}

The follows are the diagnoses of several types of faults often encountered in a power distribution system.

Scenario 1: Suppose that a fault occurs on $d_{4}$ at the terminal T-point. The detected currents $I_{3}$ and $I_{4}$ on $\mathrm{FTU}_{3}$ and $\mathrm{FTU}_{4}$ should then be set to 1 according to Equation (3), and the actual states uploaded 
by FTUs are $I_{j}=\left[\begin{array}{llllll}0 & 0 & 1 & 1 & 0 & 0\end{array}\right]$. The corresponding objective function and the functions computing the expected state of the sectionalizing switches are constructed using the analytic model of the upper level. The candidate FHs are obtained and sorted in Table 4.

Table 4. Diagnoses using the upper-level analytic model in scenario 1 and 2.

\begin{tabular}{ccccc}
\hline No. & Candidate FHs & Fault Components & Missing Alarm on FTU & False Alarm on FTU \\
\hline 1 & 000100000000000000000 & $d_{4}$ & $/$ & $/$ \\
2 & 000010000001000000000 & $d_{5}$ & $S_{5}$ & $/$ \\
3 & 000000100000010000000 & $d_{7}$ & $S_{7}$ & $/$ \\
3 & 001000000000000001000 & $d_{3}$ & $/$ & $S_{4}$ \\
4 & 000000000000000011000 & $/$ & $/$ & $S_{3}, S_{4}$ \\
\hline
\end{tabular}

A three-phase metal grounding fault occurred in the middle of $d_{4}$ is simulated using GRS. The maximum load current is $600 \mathrm{~A}$, thus the value of $I_{\mathrm{Jzd}}$ should be set to more than $600 \mathrm{~A}$. The fault currents are calculated as $127 \mathrm{~A}$ on $\mathrm{FTU}_{1}, 2732 \mathrm{~A}$ on $\mathrm{FTU}_{3}, 2732 \mathrm{~A}$ on $\mathrm{FTU}_{4}$, and $527 \mathrm{~A}_{\text {on FTU }}$. The fault does not occur on the corresponding terminal T-point where $S_{1}$ locates because the fault current on $\mathrm{FTU}_{1}$ is less than $I_{\mathrm{Jzd}}$. Fault overcurrent is monitored on $S_{4}$, and the fault current magnitudes on $S_{7}$ and $S_{5}$ do not match the abrupt-variation criterion, with the threshold set as $2 I_{\mathrm{Jzd}}$. Therefore, the preliminary diagnosis result of the fault section is $d_{4}$.

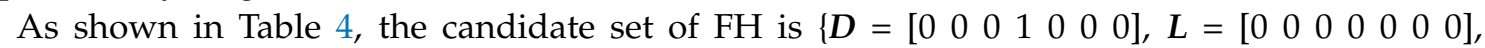
$W=\left[\begin{array}{llllll}0 & 0 & 0 & 0 & 0 & 0\end{array}\right]$. So, the final diagnosed fault location is on $d_{4}$ with no missing/false alarms for all the FTUs.

Scenario 2: Suppose that a fault occurs in the middle of $d_{3}$ and the $\mathrm{FTU}_{4}$ uploads a false alarm 1. In this situation, according to Equation (3), only the current monitored on $S_{3}$ should be 1 . Because of the false alarm from $\mathrm{FTU}_{4}$, however, $I_{j}=\left[\begin{array}{llllll}0 & 0 & 1 & 1 & 0 & 0\end{array}\right]$, the same as that of Scenario 1 . As a result, the analytic mode leads to the same diagnosis (Table 4).

As having done in Scenario 1, a three-phase metal grounding fault occurred in the middle of $d_{3}$ is simulated. The fault currents are calculated as $225 \mathrm{~A}_{\text {on FTU }}, 4818 \mathrm{~A}$ on FTU $\mathrm{FT}_{3}, 488 \mathrm{~A}^{\text {on FTU }}$, and $488 \mathrm{~A}_{\text {on }} \mathrm{FTU}_{7}$, and the value of $I_{\mathrm{Jzd}}$ is set to a larger value than the largest load current $600 \mathrm{~A}$. Since the fault currents detected by $\mathrm{FTU}_{1}$ and $\mathrm{FTU}_{4}$ are both less than $I_{\mathrm{Jzd}}$, there should be no fault at the terminal T-point. However, the calculated absolute value ratio between the currents on $\mathrm{FTU}_{1}$ and $\mathrm{FTU}_{4}$ respects Equation (7), thus the preliminary diagnosis result of the fault location is $d_{3}$.

$$
\left|\frac{I_{d 3 k-1}}{I_{d 3 k}}\right|>8
$$

The matched candidate FH is found using Table 4 to get $\left\{\boldsymbol{D}=\left[\begin{array}{lllllll}0 & 0 & 1 & 0 & 0 & 0 & 0\end{array}\right], \boldsymbol{L}=\left[\begin{array}{lllllll}0 & 0 & 0 & 0 & 0 & 0 & 0\end{array}\right]\right.$,

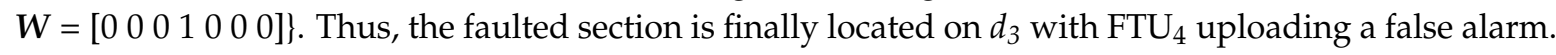

In this situation, the fault information is not correctly reflected by the "fault judgment matrix" adopted in References $[15,16]$, which will lead to a misleading diagnosis result.

Scenario 3: Suppose that the upstream FTU of the faulted section fails to report the fault information. For example: a fault occurs on $d_{4}$, whereas the missing alarm occurs on $\mathrm{FTU}_{3}$. With the critical fault information missed in this case, i.e., $I_{j}=\left[\begin{array}{lllllll}0 & 0 & 0 & 1 & 0 & 0 & 0\end{array}\right]$, the diagnosis result of the analytic model is shown in Table 5. The FH for a fault occurring on $d_{4}$ with missing alarms on $\mathrm{FTU}_{3}$ is ranked as the first among all the candidates. The fault current calculation at the lower level is the same as that of Scenario 1. After performing the two-level diagnosis, the fault is located at $d_{4}$ even though $\mathrm{FTU}_{3}$ has failed to set an alarm.

When a large number of critical fault information is missing or incorrect, it may be unable to determine the exact faulted location. Some different methods could be used in those scenarios [22-24]. For some cases where the protection system is reliable with complete alarms, it is appropriate to formulate the fault diagnosis as an optimization problem to minimize the discrepancy between the actual and expected alarms. In other cases when the alarms are accurate but incomplete, the minimum 
cover set index can be taken as the objective function of the optimization. In still others, when a large number of critical alarms is false or missing, the fault diagnosis should employ multi-source fault information to reduce the impacts brought about by the false and/or missing alarms. In any case, it is worth studying the fault diagnosis with insufficient information, especially when the false/missing alarms are crucial.

Table 5. Diagnosis using the upper-level analytic model in scenario 3.

\begin{tabular}{ccccc}
\hline No. & Candidate FHs & Fault Components & Missing Alarm on FTU & False Alarm on FTU \\
\hline 1 & 000100000100000000000 & $d_{4}$ & $S_{3}$ & $/$ \\
2 & 000000100100010000000 & $d_{7}$ & $S_{3}, S_{7}$ & $/$ \\
2 & 000010000101000000000 & $d_{5}$ & $S_{3}, S_{5}$ & $/$ \\
3 & 100000010000000001000 & $d_{1}$ & $S_{1}$ & $S_{4}$ \\
3 & 001000000100000001000 & $d_{3}$ & $S_{3}$ & $S_{4}$ \\
4 & 000000000000000001000 & $/$ & $/$ & $S_{4}$ \\
\hline
\end{tabular}

Scenario 4: A large number of alarms are missing or incorrect. As shown in Figure 3, where a fault occurs on $d_{4}$ with false alarms on $\mathrm{FTU}_{1}$ and $\mathrm{FTU}_{5}$, and the alarm of $\mathrm{FTU}_{3}$ is missing, i.e., three out of seven FTUs are not working properly. In this fault scenario, the uploaded states are $I_{j}=\left[\begin{array}{lllllll}1 & 0 & 0 & 1 & 1 & 0 & 0\end{array}\right]$ while it is supposed to be $\left[\begin{array}{llllll}0 & 0 & 1 & 1 & 0 & 0\end{array}\right]$ should all the FTUs be in normal operation. The sorted diagnosis results are shown in Table 6.

Table 6. Diagnosis using the upper-level analytic model in scenario 4.

\begin{tabular}{ccccc}
\hline No. & Candidate FHs & Fault Components & Missing Alarm on FTU & False Alarm on FTU \\
\hline 1 & 000010000100001000000 & $d_{5}$ & $S_{3}$ & $S_{1}$ \\
2 & 100000000000000001100 & $d_{1}$ & $/$ & $S_{4}, S_{5}$ \\
3 & 010000001000000001100 & $d_{2}$ & $S_{2}$ & $S_{4}, S_{5}$ \\
3 & 000100000100001000100 & $d_{4}$ & $S_{3}$ & $S_{1}, S_{5}$ \\
3 & 000001000000100001100 & $d_{6}$ & $S_{6}$ & $S_{4}, S_{5}$ \\
\hline
\end{tabular}

The actual fault is covered by the candidate FHs, as given by the fourth row of Table 6, demonstrating that the proposed method can even handle fault diagnoses in such a complicated environment.

Whenever both the telecommunication and the telemetering data are missing at the same time, some other data acquisition systems installed in the distribution system could be employed to obtain the alarm messages for the fault diagnosis. The alternative devices include remote terminal units (RTUs), distribution terminal units (DTUs), and three-phase current transformers, to name a few.

\section{Conclusions}

Existing fault diagnosis methods based on communication systems rarely consider the uncertainties presented by FTUs. Furthermore, the influence of DGs connected to a distribution system presents new challenges in identifying the fault location. Considering these factors, a two-level model for locating faults in a distribution system with DGs is developed. An analytic model to determine the faulted section is first built up at the upper level with the objective function being defined as the discrepancy between the actual states of sectionalizing switches and the expected states calculated according to the fault hypothesis and the potential false and/or missing alarms. GA is used to search for all the reasonable candidate FHs. Based on the telemetering data, a lower level model is then built up to find the FH from the candidates that best explains the received alarms. The two-level model presented can not only diagnose the faulted section(s), but also identify the incorrect and missing alarms. Both telecommunication and telemetering data are used in the two-level model, thus the accuracy of fault diagnosis could be promoted for the situations with distorted and/or missed alarms, compared with the traditional matrix-based algorithms with only telecommunication 
data employed. Finally, the feasibility and fault tolerance capability of the developed model are demonstrated by case studies with various fault scenarios.

Author Contributions: G.S., Y.Z., and C.W. proposed the methodological framework and mathematical model, and performed the simulations; H.Q., M.A.S., and L.W. designed the algorithm, reviewed, and polished the manuscript; F.W. organized the research team, and reviewed and improved the methodological framework and implementation algorithm; B.Y. and J.C. analyzed the results, reviewed the manuscript, and provided suggestions. All authors discussed the simulation results and agreed for submission.

Funding: This work is supported by National Key Research and Development Program of China (2017YFB0902900).

Conflicts of Interest: The authors declare no conflict of interest.

\section{References}

1. Baran, M.E.; El-Markaby, I. Fault analysis on distribution feeders with distributed generators. IEEE Trans. Power Syst. 2005, 20, 1757-1764. [CrossRef]

2. Naiem, A.F.; Hegazy, Y.; Abdelaziz, A.Y.; Elsharkawy, M.A. A classification technique for recloser-fuse coordination in distribution systems with distributed generation. IEEE Trans. Power Deliv. 2012, 27, 176-184. [CrossRef]

3. Dewadasa, M.; Ghosh, A.; Ledwich, G.; Wishart, M. Fault isolation in distributed generation connected distribution networks. IET Gener. Transm. Distrib. 2011, 5, 1053-1061. [CrossRef]

4. Edwards, C.J.; Davidson, E.M.; McArthur, S.D.J.; Watt, I.; Cumming, T. Flexible model-based alarm processing for protection performance assessment and incident identification. IEEE Trans. Power Syst. 2013, 28, 2584-2591. [CrossRef]

5. Ou, T.C. Ground fault current analysis with a direct building algorithm for microgrid distribution. Int. J. Electr. Power Energy Syst. 2013, 53, 867-875. [CrossRef]

6. Ou, T.C. A novel unsymmetrical faults analysis for microgrid distribution systems. Int. J. Electr. Power Energy Syst. 2012, 43, 1017-1024. [CrossRef]

7. Chen, W. Fault Section Estimation Using Fuzzy Matrix-Based Reasoning Methods. IEEE Trans. Power Deliv. 2011, 26, 205-213. [CrossRef]

8. Dos Santos Fonseca, W.A.; Bezerra, U.H.; Nunes, M.V.A.; Barros, F.G.N.; Moutinho, J.A.P. Simultaneous Fault Section Estimation and Protective Device Failure Detection Using Percentage Values of the Protective Devices Alarms. IEEE Trans. Power Syst. 2013, 28, 170-180. [CrossRef]

9. Zhu, J.; Lubkeman, D.L.; Girgis, A.A. Automated fault location and diagnosis on electric power distribution feeders. IEEE Trans. Power Deliv. 1997, 12, 801-809.

10. Senger, E.C.; Manassero, G., Jr.; Goldemberg, C.; Pellini, E.L. Automated Fault Location System for Primary Distribution Networks. IEEE Trans. Power Deliv. 2005, 20, 1332-1340. [CrossRef]

11. Lee, S.-J.; Choi, M.-S.; Kang, S.-H.; Jin, B.-G.; Lee, D.-S.; Ahn, B.-S.; Yoon, N.-S.; Kim, H.-Y.; Wee, S.-B. An intelligent and efficient fault location and diagnosis scheme for radial distribution systems. IEEE Trans. Power Deliv. 2004, 19, 524-532. [CrossRef]

12. Choi, M.-S.; Lee, S.-J.; Lee, D.-S.; Jin, B.-G. A new fault location algorithm using direct circuit analysis for distribution systems. IEEE Trans. Power Deliv. 2004, 19, 35-41. [CrossRef]

13. Salim, R.H.; de Oliveira, K.; Filomena, A.D.; Resener, M.; Bretas, A.S. Hybrid Fault Diagnosis Scheme Implementation for Power Distribution Systems Automation. IEEE Trans. Power Deliv. 2008, 23, 1846-1856. [CrossRef]

14. Brahma, S.M. Fault location in power distribution system with penetration of distributed generation. IEEE Trans. Power Deliv. 2011, 26, 1545-1553. [CrossRef]

15. Wu, L.P.; Huang, C.; Qi, Y.; Zhao, W.; Jiang, H.T. A new adaptive matrix algorithm for fault location in distribution network with distributed generation. In Proceedings of the International Conference on Electrical and Control Engineering, Yichang, China, 16-18 September 2011; pp. 499-504.

16. Yuan, C.; Zeng, X.J.; Xia, Y.F. Improved algorithm for faulted section location in distribution network with distributed generations. In Proceedings of the International Conference on Intelligent Computation Technology and Automation, Changsha, China, 20-22 October 2008; pp. 893-896. 
17. IEEE Application Guide for IEEE Std 1547, IEEE Standard for Interconnecting Distributed Resources with Electric Power Systems, in IEEE Std 1547.2-2008. 15 April 2009, pp. 1-217. Available online: http:/ /ieeexplore. ieee.org/servlet/opac?punumber=4816076 (accessed on 2 June 2018).

18. Guo, W.X.; Wen, F.S.; Liao, Z.W.; Xin, J.B. An analytic model-based approach for power system alarm processing employing temporal constraint network. IEEE Trans. Power Deliv. 2010, 25, 2435-2447. [CrossRef]

19. Wei, L.H.; Guo, W.X.; Wen, F.S.; Ledwich, G.; Liao, Z.W.; Xin, J.B. An online intelligent alarm processing system for digital substations. IEEE Trans. Power Deliv. 2011, 26, 1615-1624.

20. Ko, Y.S.; Kang, T.K.; Park, H.Y.; Kim, H.Y.; Nam, H.S. The FRTU-based fault-zone isolation method in the distribution systems. IEEE Trans. Power Deliv. 2010, 25, 1003-1009. [CrossRef]

21. Mei, N.; Shi, D.Y.; Yang, Z.L.; Duan, X.Z. A matrix-based faulted section estimation algorithm for complex distribution systems. In Proceedings of the 42nd International Universities Power Engineering Conference, Brighton, UK, 4-6 September 2007; pp. 283-289.

22. Guo, W.X.; Wen, F.S.; Ledwich, G.; Liao, Z.W.; He, X.Z.; Liang, J.H. An analytic model for fault diagnosis in power systems considering malfunctions of protective relays and circuit breakers. IEEE Trans. Power Deliv. 2010, 25, 1393-1401. [CrossRef]

23. Zhang, Y.; Zhang, Y.; Wen, F.S.; Chung, C.Y.; Tseng, C.-L.; Zhang, X.Y.; Zeng, F.; Peng, Z.Q. A fuzzy Petri net based approach for power system fault diagnosis enhanced with temporal constraints. Int. J. Electr. Power Energy Syst. 2016, 78, 215-224. [CrossRef]

24. Zhang, Y.; Chung, C.Y.; Wen, F.S.; Zhong, J.Y. An analytic model for fault diagnosis in power systems utilizing redundancy and temporal information of alarm messages. IEEE Trans. Power Syst. 2016, 31, 4877-4886. [CrossRef]

(C) 2018 by the authors. Licensee MDPI, Basel, Switzerland. This article is an open access article distributed under the terms and conditions of the Creative Commons Attribution (CC BY) license (http:/ / creativecommons.org/licenses/by/4.0/). 